\title{
Efficient Wireless Transmission of Power Using Resonators with Coupled Electric Fields
}

\author{
G. E. Leyh and M. D. Kennan, Nevada Lightning Laboratory
}

\begin{abstract}
Since its introduction by Nikola Tesla in 1900, the concept of wireless power transmission has expanded in many directions, including high-intensity microwave beams, lasers and mutual magnetic induction.

Far-field systems such as lasers, focused microwaves and large aperture RF links are well suited for traversing large $(>100 \mathrm{~m})$ distances but exhibit relatively low $(<10 \%)$ overall system efficiencies due to beam diffraction limits, atmospheric absorption and multiple conversion losses. Near-field devices using direct or resonant magnetic induction offer higher efficiencies but only at low power levels and close distances.

This paper explores the potential of using coupled electric fields between two tuned resonant transformers as a means for transmitting considerable power $(>500 \mathrm{~W})$ over laboratory-scale distances (5 to 20m) with reasonable efficiency. The paper presents design concepts, circuit simulations and test results.
\end{abstract}

Index Terms - electric fields, induction, power transmission, resonant power conversion, sparks, transformers, wireless LAN.

\section{INTRODUCTION}

$\mathrm{W}$ IRELESS power transmission systems typically fall into one of two categories; near-field and far-field designs. Near fields drop off with the square of the distance, and tend to radiate small amounts of energy. Far fields are mostly radiative, and drop off linearly with distance. By convention, two elements are considered near-field coupled when their separation is less than a few times the size of the transmitting element, and are far-field coupled when the separation is many times the transmitting element size. Several far-field wireless power prototypes have spanned kilometer-range distances.

Wireless power designs that use inductively coupled nearfields include devices such as RFID tags, electric toothbrush chargers and energy transfer systems for surgically implanted devices. Karalis et al. [1] demonstrated a system using resonant magnetic induction in 2007, capable of sending 60 watts over a distance of 2 meters. Demonstrations of far-field techniques include focused microwaves [2] (Shinohara, 1975) and lasers [3] (Landis, 1994.)

Tesla's original patent [4] resembles a far-field approach, given the large intended distance between stations compared to the station size. However, Tesla's system minimizes radiated fields and instead relies upon actual conduction, replacing the transmission line with two non-wire conductors. In this case one conductor is the Earth, and the other appears to be either a capacitive path or a direct ionized path to the ionosphere according to different descriptions of the system.
Of the designs mentioned above, the approach outlined in this paper is perhaps most similar to Tesla's system, since it does not rely upon far-field or radiated power, or magnetic coupling. However this approach differs significantly from Tesla's patented system in two important ways: A) There is no ionized path between the devices, and B) The receiver performs a synchronous detection of the received energy in order to optimize conversion efficiency. The transfer of energy in this approach occurs primarily through the electric fields between the receiver and transmitter.

\section{TeChNical Work Preparation}

While testing resonant high voltage transformers at NLL there have been several curious incidents where nearby equipment became partially energized, even though completely disconnected from any power source. The disconnected equipment would unexpectedly generate robust streamer discharges into the air or, as in a recent case, burn a grounding lead attached across the primary on a completely disconnected resonant coil system.

\section{A. Reproducing the Original Conditions}

To further characterize these undesirable energy transfers, we arranged two identical resonant air-core transformers as a transmitter and receiver. Figure 1 shows the experimental setup in the laboratory high-bay area.

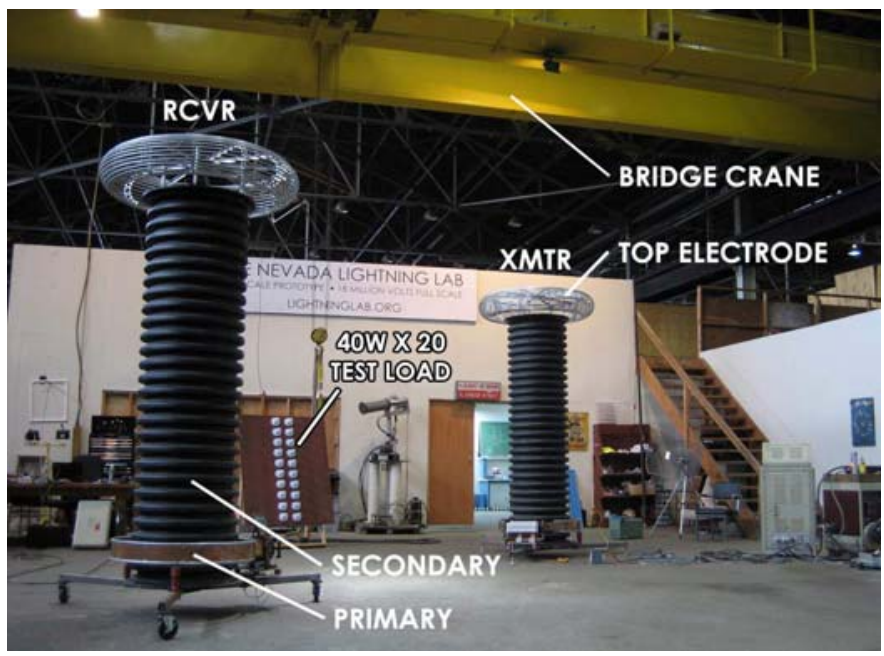

Fig. 1. Test setup with resonant transformers and 800 watt test load.

Initial tests with the transformers spaced at 5 meters yielded an unexpected power transfer of about 450 watts, measured at the resistive test load on the receiving coil. 
Further refinements to the synchronous detection circuitry in the receiving coil increased the resistive load power to 775 watts, with the transmitting coil operating at $3.6 \mathrm{~kW}$.

\section{B. Exploring Potential Coupling Mechanisms}

The first step was to isolate which coupling mechanism, or combination of coupling mechanisms, was responsible for transferring such amounts of energy between two widely separated transformers. Three possibilities were apparent:

1) Magnetic coupling between the two secondary cores

2) Electric coupling between the two top electrodes

3) Coupling through shared ground current paths

We assumed that radiated energy was negligible for this experiment, since the wavelength $(\sim 5 \mathrm{~km})$ was very large compared to the overall size of the two transformers.

The shared ground current possibility was tested first. We tested two extreme cases of grounding technique, one case where both transformers shared a long, single wire to Earth ground, and another case where each secondary had independent connections to ground, located 51 meters apart. Both cases demonstrated nearly equal levels of coupling, with the independent grounding arrangement delivering about $2 \%$ less total output to $\mathrm{R}_{\mathrm{load}}$ than the shared arrangement. This difference could be attributed in part to losses incurred by the extra lengths of grounding cable. The conclusion from this experiment was that shared ground current paths provide negligible amounts of coupling, leaving magnetic and electric fields as the two remaining possibilities.

To separately evaluate the coupling contributions provided by electric and magnetic fields we performed lumped element circuit simulations in Ansoft Simplorer, using coupling values obtained from both empirical magnetic measurements and FEM analysis of the electric fields between the top electrodes.

\section{Numerical Analysis of the Coupled Electric Fields}

To quantify the electric field coupling between the top electrodes, we developed a 3D electrostatics model using the COMSOL AC/DC module. The 3D geometry was produced in Solidworks and imported to COMSOL as a Parasolid file. To account for the steel roofing trusses and columns of the laboratory high-bay area, we set a conductive bounding box around the model that measured $15 \mathrm{~m}$ wide, $30 \mathrm{~m}$ long and $10 \mathrm{~m}$ high. The top electrode of each transformer consisted of a 11 in by 55 in aluminum space-frame toroid, and a cylindrical shielding sleeve between the coil and toroid. The COMSOL plot in Figure 2 shows the electric field pattern emanating from both toroidal top electrodes towards each other and the bounding box representing the building.

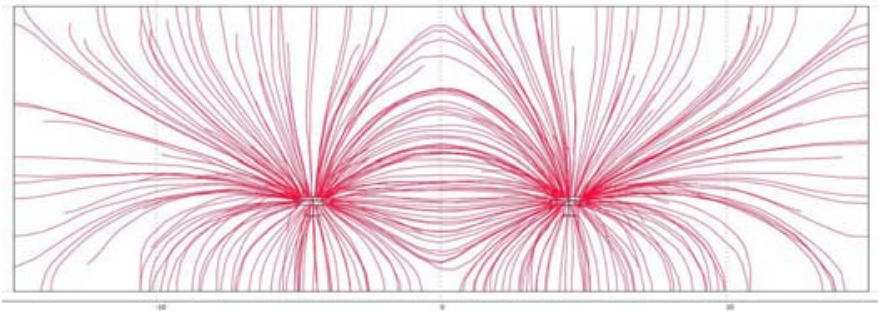

Fig. 2. 3D COMSOL electrostatics model, showing electric field pattern.
Applying fixed voltages to the top electrode surfaces, COMSOL calculated the capacitance matrix for the range of transformer separations, yielding values for the mutual capacitance vs. distance shown in Table 1, below.

\section{Empirical Measurement of the Coupled Magnetic Fields}

Real measurements of the mutual inductance for each test setup were relatively straightforward, eliminating the need for numerical analysis to determine magnetic coupling. The mutual inductance was calculated using the standard equation:

$$
V_{\text {coil }_{1}} / I_{\text {coill }_{2}}=2 * \pi * f * L_{m}
$$

Values for measured magnetic coupling and calculated mutual capacitance vs. transformer separation distance are presented in Table 1, below.

TABLE I

CALCULATED MAGNETIC AND ELECTRIC COUPLING VALUES VS. TRANSFORMER SEPARATION DISTANCE

\begin{tabular}{|c|c|c|}
\hline Separation & $\begin{array}{c}\text { k coupling } \\
\text { EMPIRICAL MSMT }\end{array}$ & $\begin{array}{c}\text { C mutual } \\
\text { COMSOL CALC }\end{array}$ \\
\hline $\mathbf{m}$ & & $\mathbf{p F}$ \\
\hline 2 & 0.003714 & 9.330 \\
3 & 0.001452 & 5.860 \\
\hline & 0.000697 & 3.710 \\
\hline 5 & 0.000394 & 2.250 \\
6 & 0.000243 & 1.360 \\
7 & 0.000183 & 0.850 \\
\hline & 0.000135 & 0.540 \\
\hline 9 & 0.000106 & 0.360 \\
\hline 10 & 0.000089 & 0.250 \\
11 & 0.000077 & 0.170 \\
\hline 12 & 0.000068 & 0.100 \\
\hline 13 & 0.000062 & 0.068 \\
\hline 14 & 0.000057 & 0.046 \\
\hline 16 & 0.000054 & 0.032 \\
\hline 17 & 0.000052 & 0.023 \\
\hline
\end{tabular}

\section{E. Circuit Simulations}

Figure 3 shows the Simplorer circuit model used for this evaluation, including the RF source, primary and secondary transmitting coils, lumped element components representing the mutual inductance and capacitance, primary and secondary receiving coils, and the resistive test load.

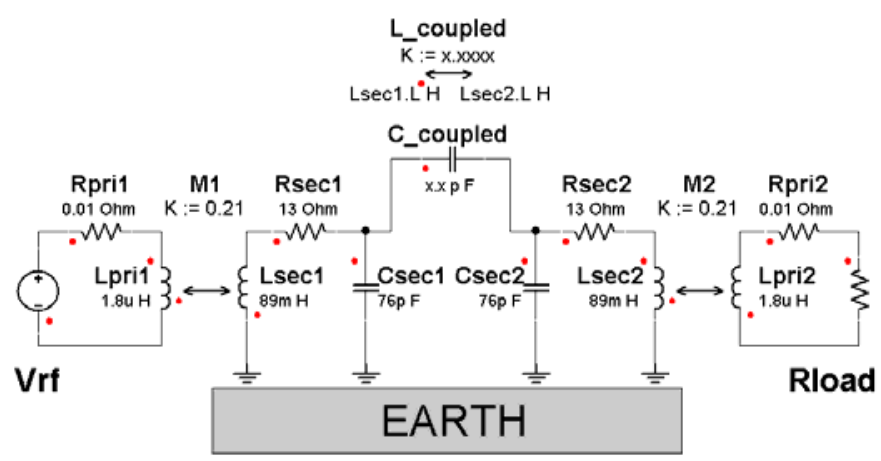

Fig. 3. Lumped element circuit model, including ambient coupling values. 
To determine the relative coupling performance provided by the mutual inductance and capacitance, Simplorer used the values in Table 1 to compute power throughput and overall efficiency for two separate cases:

a) C_coupled set to zero, L_coupled ranged from $2-17 \mathrm{~m}$

b) $\mathrm{L}_{-}$coupled set to zero, $\mathrm{C}_{-}$coupled ranged from $2-17 \mathrm{~m}$

Input power was fixed at $3.6 \mathrm{~kW}$ for all simulations. The throughput efficiency was defined as the received test load power divided by $3.6 \mathrm{~kW}$. Table 2 summarizes the Simplorer predictions for coupling performances in both cases.

TABLE 2

CALCULATED POWER THROUGHPUT AND EFFICIENCIES FOR MAGNETIC AND CAPACITIVE COUPLING VS. DISTANCE

\begin{tabular}{|c|cc|cc|}
\hline Separation & \multicolumn{2}{|c|}{ Magnetic Coupling } & \multicolumn{2}{c|}{ Capacitive Coupling } \\
& Output Power & Throughput Eff. & Output Power & Throughput Eff. \\
\hline m & W & $\%$ & W & $\%$ \\
\hline 2 & 59.18 & $1.64 \%$ & 1380.82 & $38.36 \%$ \\
3 & 10.36 & $0.29 \%$ & 1269.86 & $35.27 \%$ \\
4 & 3.21 & $0.09 \%$ & 1089.86 & $30.27 \%$ \\
5 & 1.23 & $0.03 \%$ & 838.36 & $23.29 \%$ \\
6 & $<1$ & $<0.03 \%$ & 611.51 & $16.99 \%$ \\
\hline 7 & $<1$ & $<0.03 \%$ & 404.38 & $11.23 \%$ \\
8 & $<1$ & $<0.03 \%$ & 214.52 & $5.96 \%$ \\
9 & $<1$ & $<0.03 \%$ & 115.89 & $3.22 \%$ \\
10 & $<1$ & $<0.03 \%$ & 56.71 & $1.58 \%$ \\
11 & $<1$ & $<0.03 \%$ & $<36$ & $<1 \%$ \\
12 & $<1$ & $<0.03 \%$ & $<36$ & $<1 \%$ \\
13 & $<1$ & $<0.03 \%$ & $<36$ & $<1 \%$ \\
14 & $<1$ & $<0.03 \%$ & $<36$ & $<1 \%$ \\
15 & $<1$ & $<0.03 \%$ & $<36$ & $<1 \%$ \\
16 & $<1$ & $<0.03 \%$ & $<36$ & $<1 \%$ \\
17 & $<1$ & $<0.03 \%$ & $<36$ & $<1 \%$ \\
\hline
\end{tabular}

As can be seen from the table, the circuit simulations predict that magnetic coupling contributions are relatively small, and negligible beyond a few meters range.

\section{F. Ranging Tests: Actual Throughput Power vs. Distance}

The two transformers used for measuring throughput power as a function of separation distance are shown in Figure 1. The transmitting unit was stationary, while the receiving unit was outfitted with casters to facilitate positioning. Markers on the ground at one meter increments indicated receiver range.

TABLE 3

COMPARISON OF ACTUAL RANGING TESTS VS. CAPACITIVE COUPLING SIMULATION RESULTS AT 3.6KW INPUT

\begin{tabular}{|c|c|c|c|c|}
\hline Separation & \multicolumn{2}{|c|}{ Simulation Results } & \multicolumn{2}{|c|}{ Actual Ranging Test } \\
& Output Power & Throughput Eff. & Output Power & Throughput Eff. \\
\hline $\mathbf{m}$ & $\mathbf{W}$ & $\%$ & $\mathbf{W}$ & $\mathbf{W}$ \\
\hline 2 & 1380.82 & $38.36 \%$ & $* \star \star$ & $\star \star \star$ \\
\hline 3 & 1269.86 & $35.27 \%$ & $* \star \star$ & $* \star *$ \\
\hline 4 & 1089.86 & $30.27 \%$ & $* \star$ & $22.27 \%$ \\
\hline 5 & 838.36 & $23.29 \%$ & 801.60 & $16.54 \%$ \\
\hline 6 & 611.51 & $16.99 \%$ & 595.60 & $10.32 \%$ \\
\hline 7 & 404.38 & $11.23 \%$ & 371.60 & $5.77 \%$ \\
\hline 8 & 214.52 & $5.96 \%$ & 207.60 & $2.98 \%$ \\
\hline 9 & 115.89 & $3.22 \%$ & 107.40 & $1.53 \%$ \\
\hline 10 & 56.71 & $1.58 \%$ & 55.00 & $0.75 \%$ \\
\hline 11 & $<36$ & $<1 \%$ & 27.00 & $0.34 \%$ \\
\hline 12 & $<36$ & $<1 \%$ & 12.20 & $<0.25 \%$ \\
\hline 13 & $<36$ & $<1 \%$ & $<10$ & $<0.25 \%$ \\
\hline 14 & $<36$ & $<1 \%$ & $<10$ & $<0.25 \%$ \\
\hline 15 & $<36$ & $<1 \%$ & $<10$ & $<0.25 \%$ \\
\hline 16 & $<36$ & $<1 \%$ & $<10$ & $<0.25 \%$ \\
\hline 17 & $<36$ & $<1 \%$ & $<10$ & \\
\hline
\end{tabular}

*** Not measured - Arcing hazard too great at close separation.
For each ranging test, the transmitter power was set at $3.6 \mathrm{~kW}$, to match the initial conditions in the circuit simulations. Note the absence of test data at center-to-center transformer distances of $4 \mathrm{~m}$ or less. Since each top electrode measured $1.4 \mathrm{~m}$ in diameter, the arcing hazard between the two top electrodes was too great at close separation distances.

The large experimental coil seen at the back of the highbay area in Figure 5 was grounded for these tests, otherwise it would 'rob' noticeable amounts of energy from the two test coils during the ranging tests.

As can be seen in Table 3, the actual ranging test results are in close agreement with the simulated performance predictions, based purely on electric field coupling through mutual capacitance between the two top electrodes. Figure 4 below shows this correlation in graph form.

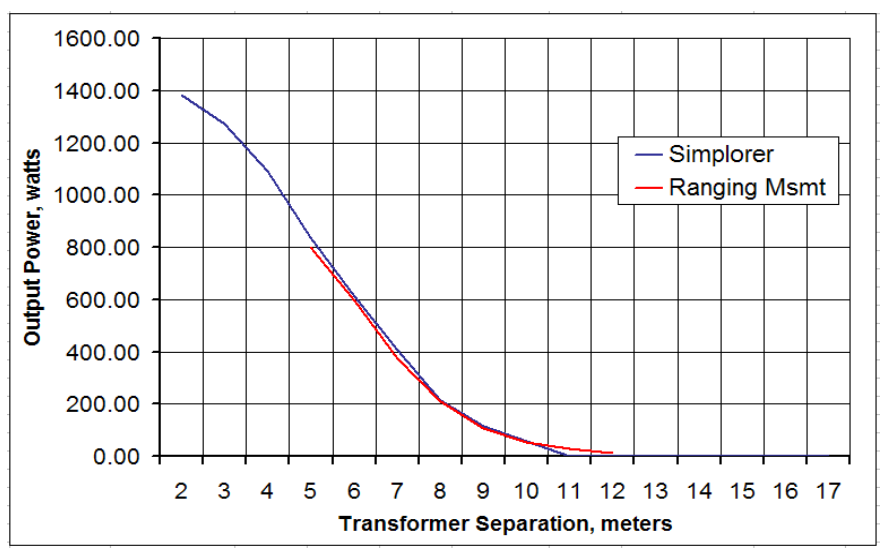

Fig. 4. Table 3 data plotted in graph format.

\section{G. Electrostatic Curtain Test}

To confirm that the coupling between the two transformers was primarily electric in nature, we constructed a movable electrostatic shielding curtain $6 \mathrm{~m}$ high by $6 \mathrm{~m}$ wide, designed to spoil electric fields while leaving magnetic fields unaffected. The curtain consisted of a horizontal steel beam supported by the overhead gantry crane, with weighted vertical wires hanging from the beam at $300 \mathrm{~mm}$ intervals. Since the wires were only electrically connected at one end to the beam, no eddy currents could flow in the curtain structure.

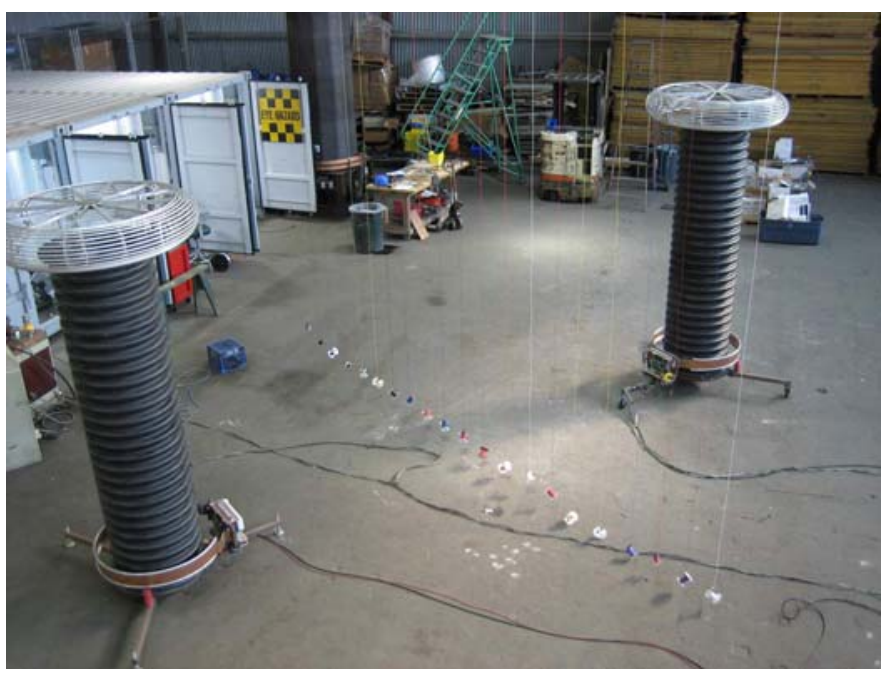

Fig. 5. Electrostatic test curtain in position between transformers. 
For the curtain test, the transformers were spaced at $6 \mathrm{~m}$. With no curtain in place the receiver coil delivered 520 watts into the load. This received power level was slightly lower than previous tests at $6 \mathrm{~m}$ for two reasons. First, the crane bridge was positioned directly over the coils at $6 \mathrm{~m}$ altitude, shunting some of the overhead electric field to ground. Also the large coil seen at the back of the high-bay in Figure 5 was ungrounded during the curtain test, allowing it to receive energy, while it was grounded during the ranging tests.

Upon craning the curtain into position between the two coils, the received power dropped from 520 watts to 28 watts. After removing the curtain once again the received power returned to 520 watts, confirming that the electric field is in fact the principal source of coupling between the two coils.

\section{H. Conclusions}

Wireless transmission of power through coupled electric fields is practical and easily demonstrated at high power levels over laboratory-scale distances.

Electric fields are far more effective than magnetic fields towards coupling energy between widely spaced resonant transformer coils.

Synchronous detection of the received energy is essential for realizing higher efficiencies, which can approach $40 \%$ at near-field distances.

Considerable care must be exercised during wireless power operation to prevent fire hazards and equipment damage, resulting from unintentional coupling of energy to nearby devices and structures.

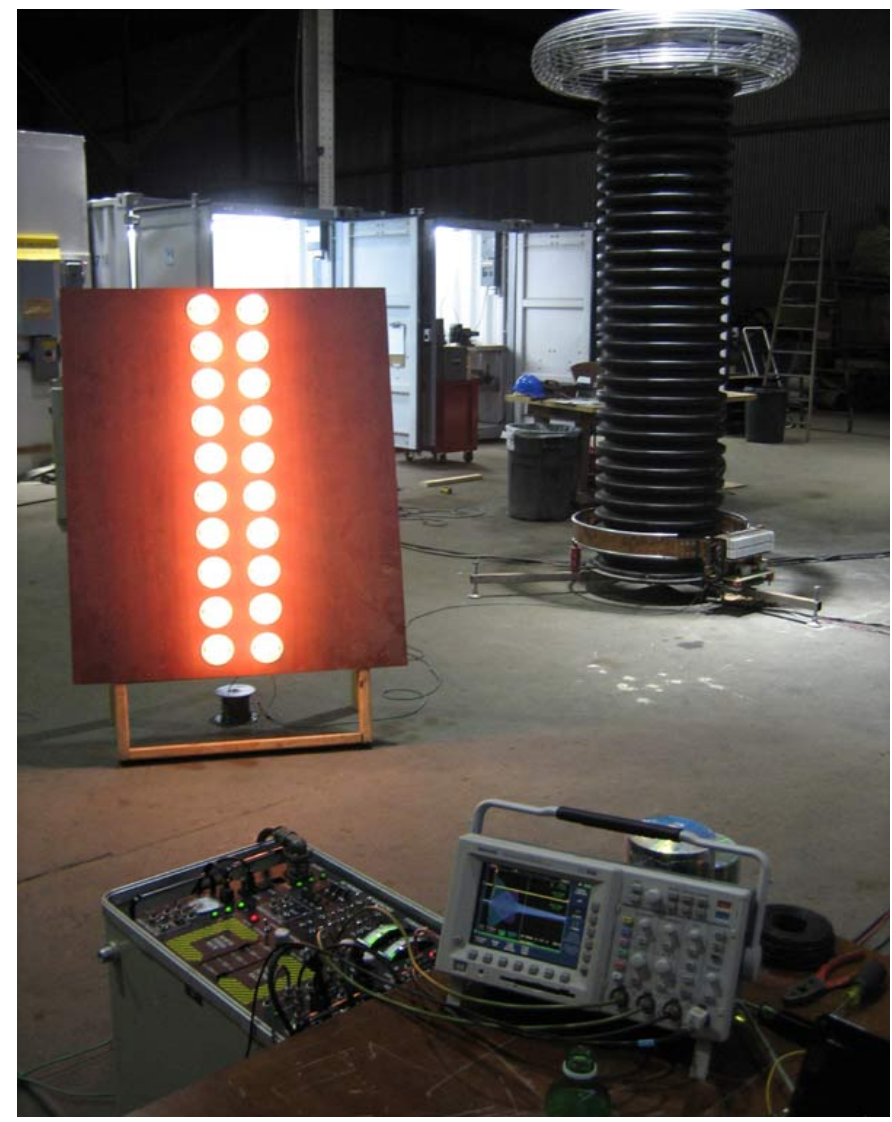

Fig. 6. Receiver coil delivering 775 watts to the $40 \mathrm{~W} \times 20$ test load.
It isn't necessary for the transmitter and receiver coils to be of identical size and shape, or even exactly the same frequency to transfer useful amounts of power. The large experimental coil in the background of Figure 5 has a selfresonant frequency about $9 \%$ lower than the two test coils, yet it can intercept over 50 watts of power from across the lab.

We are currently developing several other proof-of-concept receiver coils, including the self-motive prototype shown in Figure 7. This unit features a fast-recovery synchronous detector feeding a large, on-board capacitor bank, producing enough low-voltage DC power to provide motive effort for the machine. The unit can move about in the area near the coils, powered completely by the electric fields. The metal wheels conduct the secondary RF ground currents to the concrete.

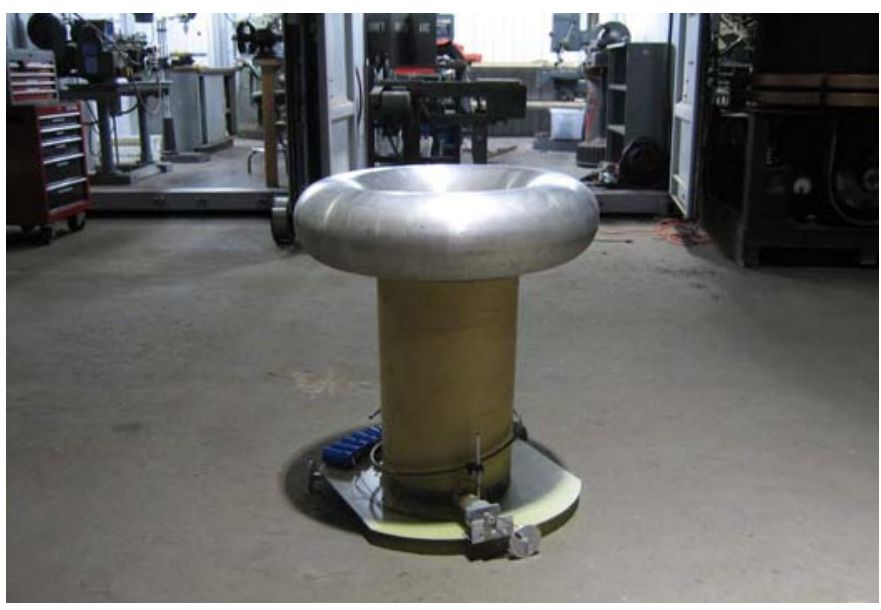

Fig. 7. Self-motive machine, powered by ambient electric fields.

\section{ACKNOWLEDGMENT}

The authors gratefully acknowledge the contributions of Brian Adolf towards consulting on the COMSOL 3D models.

\section{REFERENCES}

[1] Karalis, Aristeidis; J.D. Joannopoulos, Marin Soljačić, "Efficient wireless non-radiative mid-range energy transfer," November 2006, arXiv:physics/0611063

[2] N. Shinohara, "Wireless Power Transmission for Solar Power Satellite," JPL Goldstone Facility, 1975

[3] G. A. Landis, "Applications for Space Power by Laser Transmission," SPIE Optics, Electro-optics \& Laser Conference, Los Angeles CA, January 24-28 1994

[4] N. Tesla, "Apparatus for Transmission of Electrical Energy," U.S. Patent 649621 , May 15, 1900.

\section{BIOGRAPHIES}

Greg E. Leyh is the principal engineer for the Nevada Lightning Laboratory, a proposed research facility for exploring the large-scale physics of atmospheric electricity. He designed the Electrum Project, a $38 \mathrm{ft}$ high kinetic lightning sculpture currently operating in New Zealand.

His employment experience includes W.W. Hansen Laboratories at Stanford University, and the Stanford Linear Accelerator Center, where he developed high power IGBT designs and designed the ILC Marx Modulator for the International Linear Collider.

Mike D. Kennan is a staff engineer for the Nevada Lightning Laboratory, with over 12 years experience designing, engineering and building custom electro-mechanical systems and analog instrumentation. 